\title{
南京地区落叶栋林木本植物叶物候研究
}

\author{
朱旭斌 孙书存 $*$ \\ (南京大学生物系,南京 210093)
}

\begin{abstract}
摘 要 叶物候参数长期以来被认为与植物的碳获取的最大化有关, 能反映物种的资源利用策略。温带地区因为 寒冷冬天的限制, 延长叶寿命成为一些物种进行生长发育和繁衍的基础。为探讨叶寿命延长的可能途径( 早出叶、 晚落叶, 或两者兼有), 该研究以南京地区两个落叶柇 ( Quercus spp.) 林为研究对象, 观测了其中木本植物的出叶物 候、落叶物候, 并分析了它们与叶寿命之间的关系。结果发现: 1) 不同物种的出叶开始时间相差较大, 出叶早的物 种早结束出叶过程;2)不同物种的落叶开始时间相差较大, 早开始落叶的物种, 落叶持续时间较长, 落叶结束时间 则相对集中。3)相关分析和回归分析都表明, 叶寿命与出叶时间和落叶时间显著关联, 但早出叶对叶寿命的延长 可能更为重要, 因为早出叶相对于晚落叶在物种资源利用上比较具有优势。4)不同物种的出叶时间和落叶时间没 有显著相关, 可能因为出叶过程和落叶过程是由不同的启动因子引起。这说明延长叶寿命不一定同时通过早出叶 和晚落叶来达到。
\end{abstract}

关键词 落叶柇林 叶物候 出叶时间 落叶时间 叶寿命

\section{LEAF PHENOLOGY OF WOODY SPECIES IN DECIDUOUS BROAD-LEAVED OAK FORESTS IN NANJING AREA, EAST CHINA}

\author{
ZHU Xu-Bin and SUN Shu-Cun* \\ (Department of Biology, Nanjing University, Nanjing 210093, China)
}

\begin{abstract}
Leaf phenology has long been thought to evolve to maximize plant carbon gains, and it reflects plant life history strategies for exploiting resources. Extending leaf life span is of great importance for some species of temperate deciduous forests to gain enough carbon needed for plant survival and persistence, because their growing seasons are repeatedly interrupted by cold winters. Leaf life span can be extended by early leafing, late shedding, or both. To examine which approach was employed to maximize leaf longevity, the phenology of leaf emergence and abscission was investigated for species of two deciduous broad-leaved oak forests in Nanjing area, East China, and the relationships between leaf phenological parameters were analyzed.

The timing of leaf emergence differed greatly among species. The earlier the species leafed out, the earlier the species ended the process of leaf emergence; the earlier a species leaf began to abscise its leaves, the longer it took to end the process of leaf abscission. Consequently, the leaf abscission for all species was completed within a narrow time period although the start of leaf abscission differed greatly among species. Both correlation analysis and regression analysis indicated close relationships between leaf longevity and the timing of leaf emergence and abscission, in which a longer leaf life span was related to early leafing and late shedding. The timing of leaf emergence seems more important for plant carbon gain than the timing of leaf abscission, assuming that early leafing has advantages over late shedding for carbon gain for the woody species living in the study region. No significant relationship was found between the timing of leaf emergence and leaf abscission indicating that long leaf longevity could be achieved by either early leaf emergence or late leaf abscission. Early leafing is not necessarily correlated to late shedding for the study species.
\end{abstract}

Key words Deciduous broad-leaved oak forest, Leaf life span, Leaf phenology, Timing of leaf emergence, Timing of leaf abscission

温带地区植物的生长总是被寒冷的冬季重复地 打断, 因此植物生活史过程中的碳获取, 以及植物个 体的适合度, 受到光合作用时间的制约。这种进化
上的选择压力对于生存于逆境中的物种更为严重, 因为胁迫环境降低了植物的光合作用效率, 延长了 植物获得其完成生活史必需的有机物所需要的时间 
（Chabot \& Hicks，1982; Kikuzawa，1991; 张林和罗天 祥, 2004)。叶片是碳获取的器官, 叶物候参数, 例如 叶寿命、出叶时间和落叶时间, 能反映植物在时间上 利用资源的策略（Kikuzawa，1995）。

叶寿命已经被广泛证明是植物生活史对策的重 要参数, 一般认为叶寿命的优化会使植物的碳获取 最大化 (Chabot \& Hicks, 1982; Kikuzawa，1991; Ackerly, 1999)。已有研究发现, 立地条件, 包括土壤水 分和养分状况, 生物因子的影响, 包括竞争作用和草 食动物的取食, 都是叶寿命的决定要素 (Rathcke \& Lacey, 1985; Reich, 1993; Coley \& Barone, 1996; Westoby et al., 2002; Kikuzawa, 2003; 张林和罗天 祥, 2004)。在一个植物群落中, 因为生境异质性和 物种之间复杂的相互作用, 不同物种具有不同的碳 获取策略, 例如提高光合作用效率和降低呼吸消耗 速率, 而延长叶寿命是其中的一个可能选择。植物 有 3 种途径达到延长叶寿命的目标: 包括提早出叶, 延迟落叶,或两者兼有。不同的物种可能采取不同 途径、具有不同的出叶物候和落叶物候格局, 从而反 映植物不同的资源利用策略。例如, 早春草本植物 充分利用林冠形成前的光照资源, 在群落完全郁闭 前完成其生活史( Rathcke \& Lacey，1985); 而一些受 到草食动物侵害的植物物种则经常通过延迟落叶、 增加叶寿命来增加碳获取 (Coley \& Barone, 1996)。 在物种水平上, 不同个体之间比较发现, 早出叶的个 体, 落叶也较晚 (孙书存和陈灵芝, 1998; Seiwa, $1999 \mathrm{a}, 1999 \mathrm{~b}$ ); 多个物种的比较研究发现: 早出叶的 物种, 落叶也迟于其它物种 ( Ne' eman, 1993)。在群 落水平上, Kikuzawa $(1983,1984)$ 研究了一个落叶阔 叶林树种的叶群体存活曲线, 但没有分析出叶、落叶 物候和叶寿命之间的关系。目前群落水平上的不同 物种叶物候的比较研究仍然缺乏, 叶寿命与出叶物 候和落叶物候之间的关系尚不清晰。

本文以中国南京地区两个落叶栋林为研究对 象, 调查了群落内主要木本植物的出叶物候, 包括出 叶开始时间、结束时间和持续时间; 落叶物候, 包括 落叶开始时间、结束时间和持续时间; 以及每个物种 的叶寿命, 分析了叶寿命与出叶物候和落叶物候之 间的关系。我们假设光合作用时间是温带落叶植物 碳获取的重要限制因子, 自然选择将导致物种叶寿 命的最大化, 因此预测叶寿命长的物种出叶较早、落 叶较晚, 并且出叶较早的物种落叶较晚。具体研究 目标包括: 1) 植物出叶物候格局, 2) 植物的落叶物候
格局, 和 3)植物出叶、落叶物候与叶寿命间的关系。

\section{1 研究方法}

\section{1 研究地概况}

研究地位于南京市郊, 共两处。其中一处样地 在紫金山南坡, 位于藏经阁; 另一处位于南京市浦口 区龙王山东坡。本地区地处北亚热带, 季风明显、温 暖湿润、四季分明。年平均降水量约为 $1070 \mathrm{~mm}$, 干 燥度为 $0.97 ; 1.7$ 月平均温度分别为 $2.2 、 27.8{ }^{\circ} \mathrm{C}$; 日照资源比较丰富。土壤为黄棕壤。

本研究所选取的调查对象都是较为典型、人为 干扰较少的落叶栋林。藏经阁样地, 位于 $118^{\circ} 43^{\prime}$ $\mathrm{E}, 32^{\circ} 04^{\prime} \mathrm{N}$; 海拔 $93 \mathrm{~m}$, 群落郁闭度为 0.65 , 林冠层 主要树种有白栋 ( Quercus fabri)、枫香 (Liquidambar formosana)、栓皮栋 ( Quercus variabilis), 平均高度分别 为 $15.25 、 18.6 、 19.43 \mathrm{~m}$, 平均胸径为 $21.5 、 22.4$ 、 $35.57 \mathrm{~cm}$, 林冠层还伴生有梧桐 (Firmiana simplex)。 林下层主要树种有三角枫 ( Acer buergerianum)、英 $\square$ ( Viburnum dilatatum)、卫矛( Euonymus alatus)、木莓 (Rubus swinhoei)、糙叶树 (Aphananthe aspera)、茶条槭 (Acer ginnala)、白檀 (Symplocos paniculata)、盐肤木 (Rhus chinensis) 等物种。草本层主要物种有苔草 (Carex spp.)、天门冬 (Asparagus cochinchinensis) 等中 生草本植物, 总盖度 $85 \%$ 以上。

龙王山样地的落叶阔叶林位于 $118^{\circ} 51^{\prime} \mathrm{E}$, $32^{\circ} 03^{\prime} \mathrm{N}$; 海拔 $111 \mathrm{~m}$, 是由栎树 ( Querucs spp.) 和化 香 (Platycarya strobilacea) 为主要成分的次生天然林, 群落郁闭度为 0.6 , 林冠层主要树种有白栋、化香、 构树 (Broussonetia papyrifera), 平均高度分别为 15.1、 $11.5 、 13.2 \mathrm{~m}$, 平均胸径为 $23.1 、 17.5 、 18.2 \mathrm{~cm}$ 。林下 层主要树种有白檀、山胡椒 (Lindera glauca)、狭叶山 胡椒 (Lindera angustifolia)、朴树 (Celtis sinensis)、茶条 槭等物种。草本层主要物种有苔草、天门冬、繁缕 (Stellaria media) 等中生草本植物, 总盖度 $75 \%$ 以上。

\section{2 观测方法}

以两个研究地栋林中的主要木本植物为观测对 象。所调查物种紫金山 18 个, 龙王山 25 个, 总调查 物种 33 个(其中两地有相同物种 10 个), 分属于 21 科(表 1)。每个树种随机选取 3 5 个植株, 用于调 查叶物候。 2002 年 3 月上旬, 所有木本植物个体尚 未出叶之际, 应用随机枝取样法 (Mailleate, 1982), 在每个植株上选取 3 5 个树冠中部的标准枝, 用标 记带标记。然后, 根据叶的生长特点, 不定期记录每 
表 1 南京地区两个落叶栋林木本植物叶物候观测数据

Table 1 Data on leaf phenology of the woody species of two deciduous broad-leaved oak forests in Nanjing area, East China

\begin{tabular}{|c|c|c|c|c|c|c|c|c|c|c|}
\hline & 物种 Species & $\begin{array}{l}\text { 出叶开 } \\
\text { 始时间 } \\
\text { Beggi- } \\
\text { nning } \\
\text { time } \\
\text { of leaf } \\
\text { emerg- } \\
\text { ence }\end{array}$ & $\begin{array}{l}\text { 出叶结 } \\
\text { 束时间 } \\
\text { Ending } \\
\text { time of } \\
\text { leaf } \\
\text { emerg- } \\
\text { ence }\end{array}$ & $\begin{array}{l}\text { 出叶持 } \\
\text { 续时间 } \\
\text { Duration } \\
\text { of leaf } \\
\text { emerg- } \\
\text { ence } \\
\text { (d) }\end{array}$ & $\begin{array}{l}\text { 落叶开 } \\
\text { 始时间 } \\
\text { Begin- } \\
\text { ning } \\
\text { time of } \\
\text { leaf } \\
\text { abscis- } \\
\text { sion }\end{array}$ & $\begin{array}{l}\text { 落叶结 } \\
\text { 束时间 } \\
\text { Ending } \\
\text { time of } \\
\text { leaf } \\
\text { abscis- } \\
\text { sion }\end{array}$ & $\begin{array}{l}\text { 落叶持 } \\
\text { 续时间 } \\
\text { Duration } \\
\text { of leaf } \\
\text { abscis- } \\
\text { sion } \\
\text { (d) }\end{array}$ & $\begin{array}{l}\text { 出叶时 } \\
\text { 间 } \\
\text { Timing } \\
\text { of leaf } \\
\text { emerg- } \\
\text { ence }\end{array}$ & $\begin{array}{l}\text { 落叶 } \\
\text { 时间 } \\
\text { Timing } \\
\text { of leaf } \\
\text { abscis- } \\
\text { sion }\end{array}$ & $\begin{array}{l}\text { 叶寿 } \\
\text { 命 } \\
\text { Leaf } \\
\text { life } \\
\text { span } \\
\text { (d) }\end{array}$ \\
\hline 龙王山 & 菝葜 Smilax china & 24 & 42 & 18 & 270 & 286 & 16 & 33 & 278 & 245 \\
\hline \multirow[t]{17}{*}{ longwangshan } & 白栎 Quercus fabri & 18 & 35 & 17 & 248 & 280 & 32 & 27 & 264 & 238 \\
\hline & 白檀 Symplocos paniculata & 16 & 48 & 32 & 245 & 278 & 33 & 32 & 262 & 230 \\
\hline & 扁担木 Grewia biloba var. parviflora & 25 & 64 & 39 & 256 & 270 & 14 & 45 & 263 & 219 \\
\hline & 茶条棫 Acer ginnala & 7 & 35 & 28 & 238 & 273 & 35 & 21 & 256 & 235 \\
\hline & 葛藤 Pueraria lobata & 28 & 42 & 14 & 235 & 275 & 40 & 35 & 255 & 220 \\
\hline & 构树 Broussonetia papyrifera & 20 & 49 & 29 & 235 & 280 & 45 & 35 & 258 & 223 \\
\hline & 化香 Platycarya strobilacea & 20 & 49 & 29 & 228 & 273 & 45 & 35 & 251 & 216 \\
\hline & 黄檀 Dalbergia hupeana & 35 & 62 & 27 & 265 & 280 & 15 & 49 & 273 & 224 \\
\hline & 榔榆 Ulmus parvifolia & 18 & 35 & 17 & 242 & 274 & 32 & 27 & 258 & 232 \\
\hline & 茅莓 Rubus parvifolius & 0 & 20 & 20 & 239 & 274 & 35 & 10 & 257 & 247 \\
\hline & 朴树 Celtis sinensis & 16 & 42 & 26 & 239 & 286 & 47 & 29 & 263 & 234 \\
\hline & 山胡椒 Lindera glauca & 16 & 35 & 19 & 245 & 274 & 29 & 26 & 260 & 234 \\
\hline & 长叶鼠李 Rhamnus crenata & 23 & 55 & 32 & 249 & 275 & 26 & 39 & 262 & 223 \\
\hline & 狭叶山胡椒 Lindera angustifolia & 20 & 48 & 28 & 265 & 280 & 15 & 34 & 273 & 239 \\
\hline & 一叶 $\square$ Securinega suffruticosa & 20 & 42 & 22 & 228 & 272 & 44 & 31 & 250 & 219 \\
\hline & 柘树 Cudrania tricuspidata & 20 & 42 & 22 & 248 & 278 & 30 & 31 & 263 & 232 \\
\hline & 紫藤 Wisteria sinensis & 28 & 42 & 14 & 240 & 274 & 34 & 35 & 257 & 222 \\
\hline 藏经阁 & 白栋 Quercus fabri & 17 & 37 & 20 & 236 & 274 & 38 & 27 & 255 & 228 \\
\hline \multirow[t]{24}{*}{ Cangjingge } & 白檀 Symplocos paniculata & 23 & 57 & 34 & 260 & 286 & 26 & 40 & 273 & 233 \\
\hline & 糙叶树 Aphananthe aspera & 24 & 57 & 33 & 237 & 262 & 25 & 41 & 250 & 209 \\
\hline & 茶条槭 Acer ginnala & 17 & 43 & 26 & 246 & 285 & 39 & 30 & 266 & 236 \\
\hline & 刺楸 Kalopanax septemlobus & 20 & 50 & 30 & 225 & 285 & 60 & 35 & 255 & 220 \\
\hline & 豆腐柴 Premna microphylla & 31 & 62 & 31 & 225 & 264 & 39 & 47 & 245 & 198 \\
\hline & 枫香 Liquidambar formosana & 17 & 47 & 30 & 249 & 270 & 21 & 32 & 260 & 228 \\
\hline & 胡颓子 Elaeagnus pungens & 10 & 37 & 27 & 246 & 290 & 44 & 24 & 268 & 245 \\
\hline & 黄檀 Dalbergia hupeana & 31 & & & 241 & 269 & 28 & & 255 & \\
\hline & 英迷 Viburnum dilatatum & 10 & 37 & 27 & 255 & 285 & 30 & 24 & 270 & 247 \\
\hline & 榔榆 Ulmus parvifolia & 22 & 43 & 21 & 246 & 270 & 24 & 33 & 258 & 226 \\
\hline & 六月雪 Serissa serissoides & 10 & & & 269 & 291 & 22 & & 280 & \\
\hline & 白玉兰 Magnolia denudata & 20 & 43 & 23 & 240 & 273 & 33 & 32 & 257 & 225 \\
\hline & 木莓 Rhbus swinhoei & 10 & 43 & 33 & 233 & 291 & 58 & 27 & 262 & 236 \\
\hline & 三角枫 Acer buergerianum & 14 & 34 & 20 & 246 & 270 & 24 & 24 & 258 & 234 \\
\hline & 栓皮栎 Quercus variabilis & 19 & 42 & 23 & 225 & 268 & 43 & 31 & 247 & 216 \\
\hline & 卫矛 Euonymus alatus & 15 & 33 & 18 & 232 & 285 & 53 & 24 & 259 & 235 \\
\hline & 梧桐 Firmiana simplex & 40 & 65 & 25 & 246 & 270 & 24 & 53 & 258 & 206 \\
\hline & 小槐花 Desmodium caudatum & 31 & 49 & 18 & 232 & 290 & 58 & 40 & 261 & 221 \\
\hline & 小叶石楠 Photinia parvifolia & 19 & 50 & 31 & 242 & 267 & 25 & 35 & 255 & 220 \\
\hline & 盐肤木 Rhus chinensis & 24 & 50 & 26 & 232 & 288 & 56 & 37 & 260 & 223 \\
\hline & 野柿 Diospyros rhombifolia var. sylvestris & 31 & 65 & 34 & 241 & 272 & 31 & 48 & 257 & 209 \\
\hline & 一叶口 Securinega suffruticosa & 27 & 43 & 16 & 267 & 285 & 18 & 35 & 276 & 241 \\
\hline & 圆叶鼠李 Rhamnus globosa & 17 & 43 & 26 & 236 & 288 & 52 & 30 & 262 & 232 \\
\hline & 柘树 Cudrania tricuspidata & 23 & 50 & 27 & 241 & 273 & 32 & 37 & 257 & 221 \\
\hline
\end{tabular}

描述叶物候的时间是由 2002 年 3 月 1 日起的天数来表示 The timing for leaf phenology is indicated by the days since March 1, 2002 关于参数定 义,见正文 For more details, see text 
个枝条上的叶数量, 出叶早期 $2 \sim 3 \mathrm{~d} 1$ 次, 其后每周 1 次。对于较高大的乔木, 我们用 $6 \mathrm{~m}$ 铝梯到达树 冠中部进行观测。如果展叶后叶片长度达 $0.5 \mathrm{~cm}$, 即作为已形成的新叶记入叶数。当连续 3 次的观测 结果相同时, 停止对出叶物候观测。在 10 月初, 所 有木本植物尚未落叶时, 开始在原有标记的枝条上 的叶数量观测, 不定期记录每个枝条上的叶数量, 早 期每周 1 次, 后期 $3 \mathrm{~d}$ 左右 1 次。当树上叶片整个边 缘为黄色时, 即记录为已经脱落。落叶物候观测至 落叶结束止。

\section{3 数据处理}

首先将出叶期间和落叶期间的最大叶数作为最 大值，分别对不同时间上取得的叶数进行标准化。 然后定义叶物候的参数,包括出叶开始时间, 即一个 物种的平均出叶数量达到最大值的 $10 \%$, 出叶结束 时间, 即平均出叶数量达到最大值的 $90 \%$, 从出叶 开始至结束之间的时间为出叶持续时间; 同样对于 落叶物候中的落叶开始时间、结束时间和持续时间 也分别取 $10 \%$ 和 $90 \%$ 为临界值定义之。叶寿命的 确定是以 $50 \%$ 的出叶到 $50 \%$ 的落叶之间的时间长 度为标准, $50 \%$ 的出叶和 $50 \%$ 的落叶所在的时间被 分别定义为一个物种的出叶时间和落叶时间。

对数据正态分布的检验发现叶物候参数的数据 分布都是非正态分布, 因此 Mann-Whitney U 检验被 用来比较两个样地的叶物候参数, 以及样地内部林 冠层与林下层物种 (物种名称见样地描述)的叶物候 参数的差异。因为两样地间各种叶物候参数无显著 差异, 两样地都是典型的温带落叶栋林, 群落结构和 性质相似, 考虑每个样地的观测物种数量有限, 我们 将两个样地的数据进行了合并。本研究中所有叶物 候和叶寿命关系等的描述和分析都建立在数据合并 的基础上。不同参数间的相关性采用 Spearman 等 级相关的非参数相关分析检验, 叶寿命与叶物候参 数的关系应用最小二乘法的线性回归分析。

\section{2 结 果}

\section{1 出叶物候}

两个栎林中木本植物的萌动时间集中在 3 月中 旬至 4 月初。不同物种的出叶开始时间差异很大 (表 1): 茅莓(Rubus parviflorius) 最早在 3 月初出叶, 比出叶最晚的梧桐早 $40 \mathrm{~d}$ 。但大部分物种都集中在 3 月 20 日左右, $70 \%$ 以上的物种出叶开始时间在 3 月 15 到 25 日之间。大部分物种出叶结束时间在 4 月中旬, 但不同物种出叶结束时间仍然相差较大, 茅
莓在 3 月 20 日已经完成出叶过程, 这时很多物种还 没有开始出叶; 而最晚结束出叶的野柿 (Diospyros rhombifolia var. sylvestris), 到 5 月初才结束出叶过 程, 两者相差达 $45 \mathrm{~d}$ 。主要木本植物的出叶持续时 间在 14 到 $33 \mathrm{~d}$ 之间, 平均出叶持续时间为 $25 \mathrm{~d}$, 因 此植物的出叶过程相对集中。

不同物种间的比较表明 (表 2, 图 1), 物种的出 叶开始越早, 结束也较早; 而且持续时间越长, 结束 出叶过程就越晚; 出叶开始时间与持续时间无关。 这一定程度上说明早出叶的物种并没有降低其出叶 速度,而是较出叶开始较晚的物种先完成出叶过程。
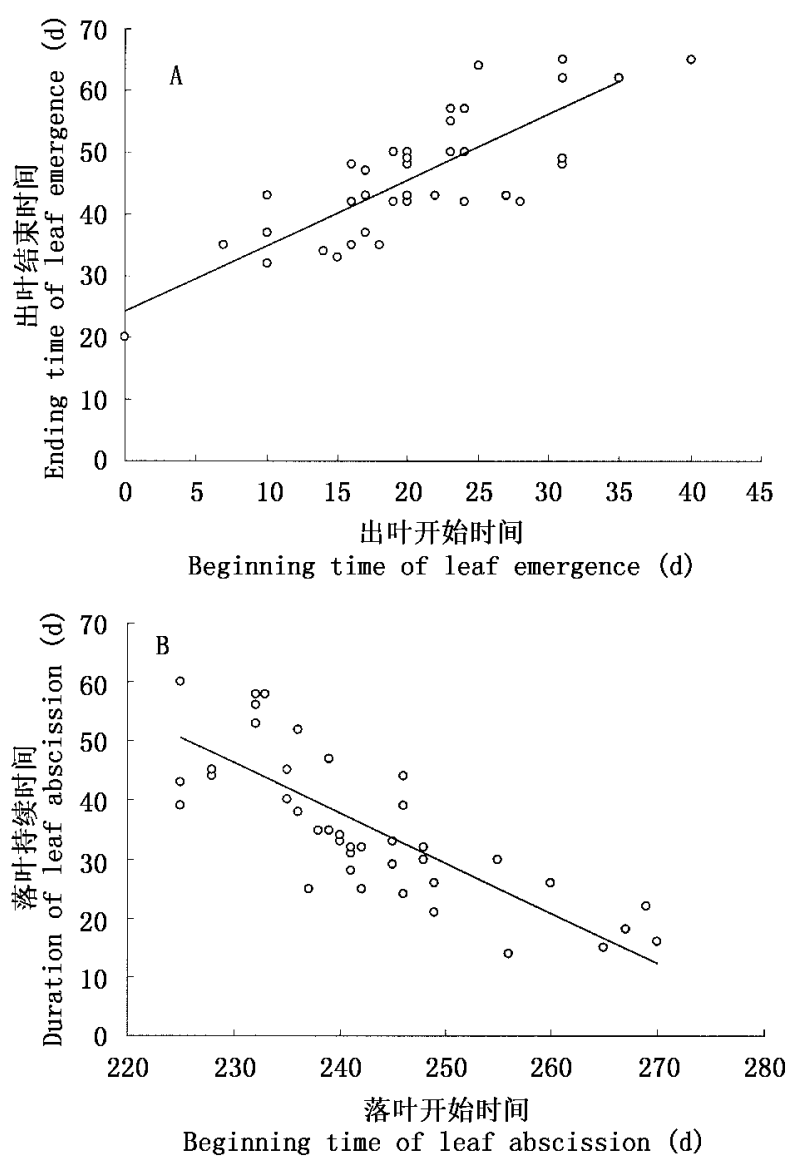

图 1 出叶物候和落叶物候中参数间关系

Fig. 1 The relationships between phenological parameters of leaf emergence and leaf abscission

A: 出叶物候中, 出叶开始时间与出叶结束时间关系 The beginning time and the ending time of leaf emergence $\left(R^{2}=0.604, p<0.001\right)$ $\mathrm{B}$ : 落叶物候中, 落叶开始时间与落叶持续时间关系 The beginning time of leaf abscission and the duration of leaf abscission $\left(R^{2}=0.626, p<\right.$ 0.001）图中描述叶物候的时间是由 2002 年 3 月 1 日起的天数 The timing for leaf phenology is defined as the days since March 1, 2002

\section{2 落叶物候}

两个楿林开始落叶时间最早的物种都在 10 月 中旬, 大部分物种在 11 月中旬开始落叶。不同物种 开始落叶时间相差很大 (表1), 从最早开始落叶的 
表 2 南京地区落叶栋林叶物候参数的相关分析

Table 2 Correlation analysis for leaf phenological parameters of the woody species of deciduous broad-leaved oak forests in Nanjing area, East China

\begin{tabular}{|c|c|c|c|c|c|c|c|c|}
\hline & $\begin{array}{l}\text { 出叶开始 } \\
\text { 时间 } \\
\text { Begginning } \\
\text { time of leaf } \\
\text { emergence }\end{array}$ & $\begin{array}{l}\text { 出叶结束 } \\
\text { 时间 } \\
\text { Ending time } \\
\text { of leaf e- } \\
\text { mergence }\end{array}$ & $\begin{array}{l}\text { 出叶持续 } \\
\text { 时间 } \\
\text { Duration of } \\
\text { leaf emer- } \\
\text { gence (d) }\end{array}$ & $\begin{array}{l}\text { 落叶开始 } \\
\text { 时间 } \\
\text { Beginning } \\
\text { time of leaf } \\
\text { abscission }\end{array}$ & $\begin{array}{l}\text { 落叶结束 } \\
\text { 时间 } \\
\text { Ending time } \\
\text { of leaf ab- } \\
\text { scission }\end{array}$ & $\begin{array}{l}\text { 落叶持续 } \\
\text { 时间 } \\
\text { Duratioin of } \\
\text { leaf abscis- } \\
\text { sion (d) }\end{array}$ & $\begin{array}{l}\text { 出叶时间 } \\
\text { Timing of } \\
\text { leaf emer- } \\
\text { gence }\end{array}$ & $\begin{array}{l}\text { 落叶时间 } \\
\text { Timing of } \\
\text { leaf abscis- } \\
\text { sion }\end{array}$ \\
\hline $\begin{array}{l}\text { 叶寿命 } \\
\text { Leaf life span (d) }\end{array}$ & $-0.607^{* * *}$ & $-0.680^{* * *}$ & $-0.306^{\mathrm{ns}}$ & $0.490^{* * *}$ & $0.573^{* * *}$ & $-0.140^{\mathrm{ns}}$ & $-0.730^{* * *}$ & $0.653^{* * *}$ \\
\hline $\begin{array}{l}\text { 落叶时间 } \\
\text { Timing of leaf abscission }\end{array}$ & $-0.143^{\mathrm{ns}}$ & $-0.063^{\mathrm{ns}}$ & $-0.042^{\mathrm{ns}}$ & $0.757^{* * *}$ & $0.708^{* * *}$ & $-0.352^{*}$ & $-0.094^{\mathrm{ns}}$ & \\
\hline $\begin{array}{l}\text { 出叶时间 } \\
\text { Timing of leaf emergence }\end{array}$ & $0.830^{* * *}$ & $0.915^{* * *}$ & $0.352^{*}$ & $-0.013^{\text {ns }}$ & $-0.196^{\mathrm{ns}}$ & $-0.176^{\mathrm{ns}}$ & & \\
\hline $\begin{array}{l}\text { 落叶持续时间 } \\
\text { Duratioin of leaf abscission (d) }\end{array}$ & $-0.240^{\mathrm{ns}}$ & $-0.119^{\mathrm{ns}}$ & $-0.082^{\mathrm{ns}}$ & $-0.839^{* * *}$ & $0.330^{*}$ & & & \\
\hline $\begin{array}{l}\text { 落叶结束时间 } \\
\text { Ending time of leaf abscission } \\
\text { 落叶开始时间 }\end{array}$ & $-0.267^{\mathrm{ns}}$ & $-0.168^{\mathrm{ns}}$ & $-0.150^{\mathrm{ns}}$ & $0.151^{\mathrm{ns}}$ & & & & \\
\hline $\begin{array}{l}\text { Beginning time of leaf abscis- } \\
\text { sion }\end{array}$ & $0.011^{\mathrm{ns}}$ & $-0.021^{\mathrm{ns}}$ & $0.019^{\text {ns }}$ & & & & & \\
\hline $\begin{array}{l}\text { 出叶持续时间 } \\
\text { Duration of leaf emergence (d) }\end{array}$ & $0.025^{\mathrm{ns}}$ & $0.671^{* * *}$ & & & & & & \\
\hline $\begin{array}{l}\text { 出叶结束时间 } \\
\text { Ending time of leaf emergence }\end{array}$ & $0.626^{* * *}$ & & & & & & & \\
\hline
\end{tabular}

豆腐柴 ( Premna microphylla), 到最晚的菝葜 (Smilax china), 相差达 $45 \mathrm{~d}$ 。相对而言, 植物落叶结束时间 相对集中, 所有物种在 12 月初叶片即已经全部脱 落, 最早结束落叶的物种和最晚的之间相差不到 30 $\mathrm{d}$ 。落叶的持续时间相差很大, 最快的物种在 $14 \mathrm{~d}$ 内完成整个落叶过程, 刺楸 (Kalopanax septemlobus) 的落叶持续时间竞有 $60 \mathrm{~d}$ 之久。平均落叶持续时 间约 $34 \mathrm{~d}$ 。

对落叶物候参数的相关分析表明 (表 2, 图 1): 植物的开始落叶时间越早, 落叶持续周期就越长; 落 叶持续越长, 落叶结束时间越晚; 但物种的落叶开始 时间与其结束时间无显著相关。这一定程度上表 示, 早开始落叶的物种, 因为延长落叶持续时间, 降 低了与后开始落叶的物种在落叶结束时间上的差 异。

林冠层与林下层物种在所研究的叶物候参数上 均无显著差异。

\section{3 叶寿命及其与出叶、落叶物候的关系}

两个栎林木本植物的平均叶寿命为 $228 \mathrm{~d}$, 寿命 最短的为豆腐柴, 仅 $198 \mathrm{~d}$, 寿命最长的为茅莓和荚 $\square$, 达到 $247 \mathrm{~d}$, 相差近 $50 \mathrm{~d}$ 。对叶物候和叶寿命等 参数的相关分析表明 (表 2, 图 2), 叶寿命与出叶物 候和落叶物候除落叶持续时间外的参数均有显著相 关。叶寿命与出叶时间和落叶时间的回归分析表
明, 植物出叶时间越早, 或者落叶时间越晚, 叶寿命 越长。但是回归分析和相关分析都没有发现植物出 叶和落叶时间之间存在显著关系。

\section{3 讨 论}

我们的结果表明,在所调查的两个群落中,不同 物种的出叶物候和落叶物候都相差很大, 从而导致 了不同物种叶寿命之间的差异。物种之间的比较分 析说明, 叶寿命与出叶开始时间和落叶开始时间有 显著关联, 叶寿命长的物种出叶早、落叶晚, 与预测 一致。但物种的出叶时间和落叶时间并没有显著关 联, 与我们的预测不一致; 也就是说, 在群落水平上, 物种并非一定同时通过提前出叶和延迟落叶来增加 叶寿命, 与现有物种水平上研究结果是不同的。

碳获取是物种生长发育的基础, 是物种繁衍后 代, 维持物种延续的保证。生活在同一个群落中的 物种, 因为具有不同的竞争能力和不同的群落优势 地位, 它们在同一种生境中获取资源的能力发生差 异。在温带地区, 一般而言, 林冠层的建群种具有较 强的竞争能力, 能摄取分布集中、质量兼优的资源, 例如林冠上层的高大乔木依赖茎的支持可以摄取充 足阳光, 依赖粗大深根系可以提供充足的水分, 因此 具有较高的碳获取效率。但其它竞争能力弱的物 种, 虽然不能取得优质资源, 但可以通过不同的碳获 


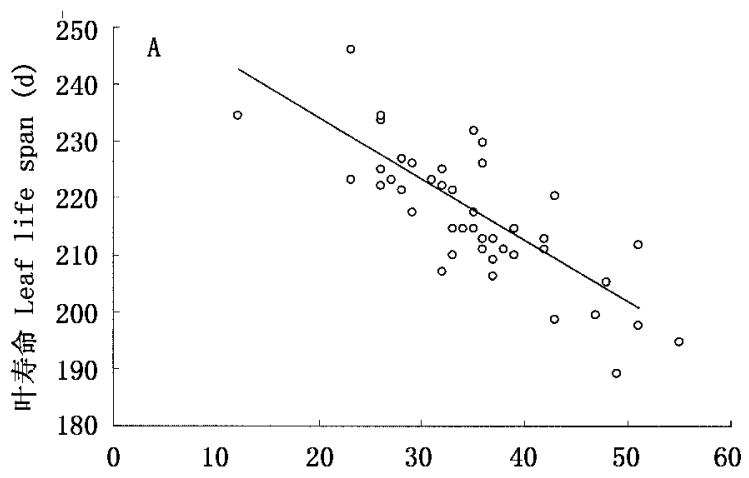

出叶时间 Timing of leaf emergence (d)

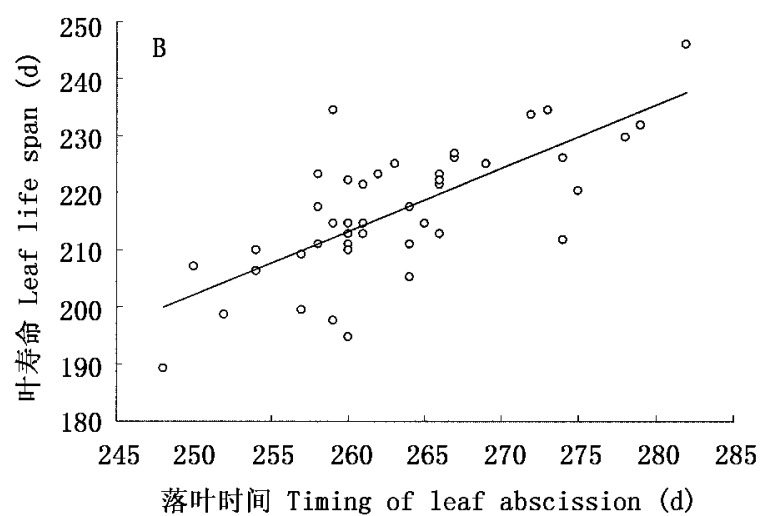

图 2 叶寿命与出叶物候、落叶物候之间的关系

Fig.2 The relationships between leaf life span and leaf phenological parameters of leaf emergence and abscission

A: 出叶物候中, 出叶开始时间与出叶结束时间关系 The beginning time and the ending time of leaf emergence $\left(R^{2}=0.552, p<0.001\right)$ $\mathrm{B}$ : 落叶物候中, 落叶开始时间与落叶持续时间关系 The beginning time of leaf abscission and the duration of leaf abscission $\left(R^{2}=0.462, p<\right.$ 0.001）图中描述叶物候的时间是由 2002 年 3 月 1 日起的天数 The timing for leaf phenology is defined as the days since March 1, 2002

取策略完成生活史过程, 如在养分贫乏、水分缺乏, 或者荫蔽的立地条件下, 增加资源的利用效率 (Westoby et al.,2002; Kikuzawa, 2003)。另一个途径 就是通过延长叶寿命增加资源利用时间, 从而提高 碳获取能力。来自不同生态系统和生物群区的物种 调查和统计分析都表明, 植物物种的最大光合作用 效率与叶寿命成反比 (Reich et al., 1992; Reich, 1993; Westoby et al., 2002), 这暗示物种可能通过 两种途径, 即提高光合作用效率或者延长叶寿命, 增 加碳获取。同样, 有很多实验研究表明, 在胁迫生境 的植物个体通常具有较长的叶寿命 (Reich, 1993; Westoby et al., 2002; Kikuzawa, 2003); 这些都提示 叶寿命是调节植物碳获取的重要因子。虽然我们没 有立地条件的比较研究, 但在同一个群落中, 不同物 种的叶寿命相差达 $50 \mathrm{~d}$, 本身就说明了生境差异的 影响, 以及不同物种可能具有不同生存策略。王希
华等(2000)曾在浙江天童常绿阔叶林发现灌木和小 乔木的叶片寿命较中乔木长, 即可能是因为不同的 光照生境影响所致。本研究中, 林冠层与林下层物 种在叶物候参数上没有显著差异。虽然出叶最早的 总是林下层物种, 叶寿命最长的也是林下层物种 (表 1 ), 但是林下层不同物种间差异很大, 部分物种较林 冠层物种出叶更晚, 叶寿命更短。例如, 一些小灌木 的叶寿命却较大型乔木短, 如豆腐柴和野柿, 它们处 于林下的荫蔽环境中, 即使能充分利用林下光斑, 在 短时间内不可能具有较多的碳获取。因此我们认为 可能有另外的生活史对策机制。改变生物量分配可 能是其中的一种, 将较多的碳用于生殖分配可以保 证其物种的延续。

提早出叶和推迟落叶都是提高叶寿命的途径。 早出叶已经被很多研究证明对于植物个体的碳获取 至关重要。在温带落叶阔叶林中的五角枫 ( Acer mono ), Seiwa（1998）发现早期萌发的幼苗在林冠郁 闭前两个月就开始出叶, 其年碳获取的 61\% 79\% 是在这段时间。而且, 他还发现 ( Seiwa, 1999a, 1999b), 五角枫和日本黑榆 (Ulmus davidiana var. japonica) 出叶顺序是, 个体高度越高, 出叶就越晚, 矮小的个体相对更能利用早春的光照资源。美国的 中西部、加拿大东部地区的林下灌木入侵种,包括金 银忍冬 (Lonicera maackii), 莫罗忍冬 (L. morrowii), 新疆忍冬 ( L. tatarica), 它们取得入侵成功并获取优 势的原因就是比当地物种提前出叶, 延长其生长季 节。Harrington 等 (1989) 发现这些物种虽然只较当 地灌木种和林冠建群种早出叶 1 周, 但在此时间内 却得到了年碳获取的 $35 \%$ 。本研究发现物种的出 叶时间与植物叶寿命成反比, 出叶越早叶寿命越长; 叶寿命与出叶结束时间成反比, 出叶结束时间越早, 叶寿命越长。实际上, 本研究中叶寿命较长的几个 物种, 如荚 $\square$ 、胡颓子(Elaeagnus pungens), 拢荰等都 是出叶时间较早和落叶时间较晚的物种。这些无疑 都表明了出叶物候对叶寿命的影响。

落叶物候在本研究中也同样与叶寿命紧密相 关, 落叶时间越晚, 叶寿命越长。然而我们认为落叶 时间对物种的碳摄取的意义与出叶时间是不一样 的, 尽管它们都与叶寿命显著相关。本文相关分析 和回归分析的结果只是在数学上表明早出叶和晚落 叶都是延长叶寿命的基本条件, 过早的落叶和过晚 出叶都不可能导致长寿命的结果, 但这并不说明早 出叶的物种就一定晚落叶, 也不能说明早出叶和晚 落叶对于物种的生态学意义同样重要。在本研究 
中, 我们没有发现一个叶寿命长的物种是通过同时 延迟出叶和落叶来达到的, 相反有些物种出叶较早, 落叶相对较早, 却也能达到相对的长寿命, 如茅莓。 其中主要原因在于群落中不同物种的出叶时间相差 较大,而落叶时间却相对集中。因为先开始出叶的 物种就早结束出叶过程 (表 2, 图 1), 物种之间的出 叶时间差异就可能很大。但大部分物种都集中在 11 月中旬落叶, 12 月 5 日所有调查的物种全部结束 了落叶过程, 这样就降低了物种在落叶时间的差异。 相反, 有些物种即使落叶开始时间较早, 但因为落叶 开始时间越早, 落叶持续时间越长(表 2, 图 1), 因此 整体上物种的叶寿命仍能得到一定程度的延长; 也 就是说群落中的不同物种虽然落叶开始时间可能相 差较大, 但落叶结束时间仍相对集中。这可能与温 带地区秋冬季节相交时的剧烈气候变化有关。这种 集中落叶现象在其它温带落叶阔叶林树种也有过记 录 (Kikuzawa，1983，1984; 孙书存和陈灵芝，1998; $\mathrm{Ne}$ ' eman, 1993)。以上分析说明, 出叶时间对一个物 种叶寿命的影响可能比落叶时间更为重要。

相对于延迟落叶, 早出叶于物种而言可能具有 更大的选择优势。首先早萌动、早出叶不仅能获取 充足的光照资源, 同时在水分和养分利用上也具有 优势。在大部分物种尚处于休眠状态, 水分和养分 利用很微弱的时候, 提早萌动即可在时间上避开与 其它物种的资源竞争, 从而保证较高的利用效率。 Hooper(1998) 等在研究一个落叶阔叶林时曾发现, 因 为物候期的差异, 不同物种通过相互作用而提高资 源利用效率, 增加群落生产力水平, 并能维持较高的 物种多样性。其次, 相对于晚落叶, 早出叶的物种对 光照资源的利用效率可能更高。叶片的光合效率随 叶片的生活史过程而变化, 在展叶过程的最早期, 叶 片的光合速率较低而呼吸作用旺盛, 随后光合效率 逐渐增加, 呼吸效率也维持在一定水平上, 但随叶片 年龄的增加, 叶片的光合作用和呼吸效率都逐渐下 降 (Gill et al., 1998; 孙书存和陈灵芝, 2000a), 直至 叶片衰老死亡。早出叶能保证叶片以较高的光合效 率进行碳获取; 而叶片衰老期间的叶寿命延长, 对于 碳获取的价值显然不能与早出叶相提并论。再次, 相对于晚落叶, 早出叶具有较小的风险。植物在春 季出叶阶段, 气温逐渐升高, 气候条件相对变得优 越,生境是可预测地向好的方向转化, 如果早出叶则 能利用到一个相对稳定的资源供应和生境; 但晚落 叶时则是面临一个不知何时降临的恶劣生境, 因此 这时植物如果作出维持或者提高叶片的光合能力的
努力, 将面临巨大风险。因此,也可以认为本研究所 得到的两个落叶柇林的叶物候格局及其与叶寿命的 关系, 一定程度上是群落和物种对特定气候特征长 期适应的结果。我国温带地区的这种气候格局可能 使物种更倾向于调整出叶时间对环境变化作出响 应。

本研究中, 落叶时间与出叶时间并没有发现任 何关联, 与我们预测的早出叶的物种晚落叶是不一 致的。这除了从以上早出叶和延迟落叶对碳获取的 不同价值来解释外, 还有一些其它因素的作用。譬 如, 昆虫取食的影响。在植物展叶初期, 植物幼嫩叶 片易被昆虫取食, 植物一般通过增加次生代谢物的 生产进行防御, 也会通过补充叶片数量来降低补偿 损失 (孙书存和陈灵芝, 2000b), 使植物叶群体的年 龄结构复杂化, 从而可能延迟物种的落叶时间。而 在冬季来临前, 昆虫由于补充越冬的营养和储存食 物, 会加大进食, 植物叶片被取食也呈上升趋势 (于 晓东等,2001)。但此时叶片补偿作用有限, 受损的 叶片往往会提前凋落。另外, 从植物生理的角度看, 出叶和落叶过程可能是由不同的生态因子启动。

般认为温度, 主要是积温是出叶过程的主要影响因 子,而落叶过程一般认为是受光周期的影响 (张富 春, 1985)。

\section{参 考 文 献}

Ackerly DD (1999). Self-shading, carbon gain and leaf dynamics: a test of alternative optimality models. Oecologia, 119, $300-$ 310 .

Chabot BF, Hicks DJ (1982). The ecology of leaf life span. Annual Review of Systematics and Ecology, 13, 229 - 259.

Coley PD, Barone JA ( 1996). Herbivory and plant defenses in tropical forests. Annual Review of Ecology and Systematics, 27, $305-335$.

Gill PS, Amthor JS, Bormann FH (1998) . Leaf phenology, photosynthesis and the persistence of saplings and shrubs in a mature north hardwood forest. Tree Physiology, 18,281-289.

Harrington RA, Brown BJ, Reich PB (1989) . Ecophysiology of exotic and native shrubs in Southern, Wisconsin. Oecologia, 80, $356-367$.

Hooper DU (1998). The role of complementarity and competition in ecosystem responses to variation in plant diversity. Ecology, 79, $704-719$.

Kikuzawa K (1983). Leaf survival of woody plants in deciduous broad-leaved forests. 1. Tall trees. Canadian Journal of Botany, $61,2133-2139$.

Kikuzawa K (1984). Leaf survival of woody plants in deciduous broad-leaved forests. 2. Small trees and shrubs. Canadian 
Journal of Botany, 62, 2551 - 2556.

Kikuzawa K (1991) . A cost-benefit analysis of leaf habitat and leaf longevity of trees and their geographical pattern. American Naturalist, 138, $1250-1263$.

Kikuzawa K (1995) . Leaf phenology as an optimal strategy for carbon gain in plants. Canadian Journal of Botany, 73, $158-163$.

Kikuzawa K (2003). Phenological and morphological adaptation to high light environment in two woody and two herbaceous plant species. Functional Ecology, 17, 29 - 38 .

Maillette L (1982) . Structure dynamics of silver birch. I. The fate of bud. Journal of Applied Ecology, 19, 203 - 218 .

Ne' eman G (1993). Variation in leaf phenology and habitat in Quercus thubuensis, a Mediterranean deciduous tree. Journal of Ecology, 81, 627 - 634 .

Rathcke B, Lacey EP (1985). Phenological patterns of terrestrial plants. Annual Review of Systematics and Ecology, 16, 179 205 .

Reich PB (1993) . Reconciling apparent discrepancies among studies relating life span, structure, and function of leaves in contrasting plant life forms and climates: the blind men and elephant retold. Functional Ecology, 10, 768 - 776 .

Reich PB, Walters MB, Ellsworth DS (1992). Leaf life span in relation to leaf, plant, and stand characteristics among diverse ecosystems. Ecological Monographs, 62, 365-392.

Seiwa K (1998). Advantages of early germination for growth and survival of seedlings of Acer mono under different overstory phonologies in deciduous broad-leaved forests. Journal of Ecology, 86, $219 \sim 228$.

Seiwa K (1999a) . Changes in leaf phenology are dependent on tree height in Acer mono, a deciduous broad-leaved tree. Annals of Botany, 83, $355 \sim 361$.

Seiwa K (1999b) . Ontogenetic changes in leaf phenology of Ulmus davidiana var. japonica, a deciduous broad-leaved tree. Tree
Physiology, 19, $793-797$.

Sun SC (孙书存), Chen IZ (陈灵芝) (1998). Leaf demography of Quercus liaotungensis in Dongling Mountain. Acta Phytoecologica Sinica (植物生态学报), 22,538-544. (in Chinese with English abstract)

Sun SC (孙书存), Chen IZ (陈灵芝) (2000a). Leaf growth and photosynthesis of Quercus liaotungensis in Dongling Mountain. Acta Ecologica Sinica (生态学报), 20,212-217. (in Chinese with English abstract)

Sun SC (孙书存), Chen IZ (陈灵芝) (2000b). The ecological response of seedlings to simulated drought and defoliation in Quercus liaotungensis. Acta Ecologica Sinica (生态学报), 20, 893 - 897. (in Chinese with English abstract)

Wang XH (王希华), Zhang J (张捷), Zhang ZX (张正祥) (2000). Leaf longevity of evergreen broad-leaved species of Tiantong National Forest Park, Zhejiang Province. Acta Phytoecologica Sinica (植物生态学报), 24,625-629. (in Chinese with English abstract)

Westoby M, Falster DS, Moles AT, Vesk PA, Wright IJ (2002). Plant ecology strategies: some leading dimensions of variation between species. Annual Review of Ecology and Systematics, 33, $125-159$.

$\mathrm{Yu}$ XD (于晓东), Zhou HZ (周红章), Luo TH (罗天宏) (2001). Patterns of damage by phytophagous insects on leaves of Quercus liaotungensis. Acta Phytoecologica Sinica (植物生态学 报), 25, 553-562. (in Chinese with English abstract)

Zhang FC(张福春) (1985). Phenology (物侯学). Meteorology Press, Beijing. (in Chinese)

Zhang L (张林), Luo TX (罗天祥) (2004). Advances in ecological studies on leaf lifespan and associated leaf traits. Acta Phytoecologica Sinica (植物生态学报), 28,844-852. (in Chinese with English abstract) 